\title{
On the Possibility of Initiating the Proton-Boron Nuclear Fusion Reaction in the Plasma-Focus Device
}

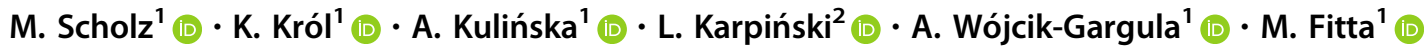

Published online: 30 August 2019

(C) The Author(s) 2019

\begin{abstract}
In this paper, the possibilities to perform aneutronic fusion in Plasma Focus (PF) devices have been presented. For this purpose, an idea of doping plasma by means of laser ablation of a solid boron target during discharge in PF-24 was presented. Some estimates have been made, based on the processes occurring during the discharge in the PF system with gas injection using a fast gas valve, indicating that this method allows the generation of hydrogen-boron plasma. The yield of the ${ }^{11} \mathrm{~B}(\mathrm{p}, \alpha) \alpha \alpha$ reaction was calculated. The results presented in this paper show that this yield is high enough to register and measure the alpha particles emitted from the plasma column consisting boron and hydrogen. The estimates were made for the parameters characteristic for PF-24.
\end{abstract}

Keywords Proton-boron reaction · Dense plasma focus · Aneutronic fusion

\section{Introduction}

The deuterium-tritium fusion reaction is foreseen as the main reaction in future controlled thermonuclear reactors in order to harvest energy. This applies to both thermonuclear fusion research based on magnetic and inertial confinement of the plasma fuel near steady-state conditions. This reaction was chosen because of its high thermal reaction rate at relatively low temperatures about $20 \mathrm{keV}$ in comparison with reactions of other light nuclei. However, a serious disadvantage of this reaction is that it is accompanied by intense high-energy neutron flux. This makes it necessary to use a thick, sophisticated blanket around the plasma as a fuel increasing considerably the size of the fusion reactor. In addition, radioactive waste is generated and tritium breeding is necessary.

Such problems can be overcome by driving the aneutronic nuclear reactions that produce substantially less high-energy radiation [1]. An example of such a reaction,

\section{A. Kulińska}

Agnieszka.Kulinska@ifj.edu.pl

1 Institute of Nuclear Physics Polish Academy of Sciences, Radzikowskiego 152, 31-342 Kraków, Poland

2 Rzeszów University of Technology, Powstańców Warszawy 12, 35-959 Rzeszów, Poland where there is almost no neutron generation, is the fusion reaction between protons $(\mathrm{p})$ and boron $\left({ }^{11} \mathrm{~B}\right)$ nuclei. Here, the energy is released as the kinetic energy of alpha particles rather than neutrons. Furthermore, the maximum of the ${ }^{11} \mathrm{~B}(\mathrm{p}, \alpha) \alpha \alpha$ cross section of 1.2 barns occurs at $600 \mathrm{keV}$ in the center of mass and it is higher than $\mathrm{d}(\mathrm{t}, \mathrm{n}){ }^{4} \mathrm{He}$ cross section for this energy. However, ${ }^{11} \mathrm{~B}(\mathrm{p}$, $\alpha) \alpha \alpha$ fusion reactivity in thermal equilibrium is too low to compete with radiation losses, thus ignition conditions cannot be achieved in this reaction [2]. It seems that this problem can be overcome by carrying out this reaction in conditions far from thermal equilibrium. Thus, various schemes were considered to implement controlled thermonuclear fusion based on the ${ }^{11} \mathrm{~B}(\mathrm{p}, \alpha) \alpha \alpha$ reaction see e.g. [3-5].

One of such systems where the fusion reaction between hydrogen and boron nuclei $\left({ }^{11} \mathrm{~B}(\mathrm{p}, \alpha) \alpha \alpha\right)$ could be carried out in conditions far from thermal equilibrium is the Plasma-Focus device (PF) [6].

In order to implement this reaction in the PF, the hydrogen-boron plasma should be created and compressed to the pinch phase, while maintaining the standard operating conditions of the PF device. This means, that as a result of the standard evolution of a discharge in the $\mathrm{PF}$, a well-compressed the hydrogen-boron plasma column will be obtained at the axis of the anode. This can be achieved 
by adding boron to the PF system at a suitably selected moment. However, it should be done in a way that will not affect the sliding electrical discharge along the PF insulator in hydrogen, which should lead to a well-formed PF current layer. A good solution to this problem seems to be the decoupling a final phase of the PF discharge from the initial one. A commonly used method of modifying a composition of plasma and its density in a compressed plasma column is the gas injection method through a high-speed electromagnetic valve located in the PF anode [7-9] or the installation of a coaxial liner on the anode front [10]. An interesting solution was also to use a dust cloud instead of gas injection in the area in front of the internal electrode [11].

A gas puffing is the classical method of change composition of plasma and increasing its density near the face of the anode plate in the PF device. This method relies on the usage of an electromagnetic valve inside the PF anode. This valve is synchronized with the PF discharge and injects gas along the anode axis to change the initial working gas with relatively low density near the anode axis of PF. A complicated technical design of the valve and placing it into the PF anode is a disadvantage of this method. Moreover, the method is limited to materials that are in a gaseous state under normal conditions. To use materials which do not occur as a gas under normal conditions, the foam liner or dust clouds was proposed to use in the PF device $[10,11]$. However, there is a limitation on the liner density, which far exceeds that of the current sheath even when the PF discharge is initiated in a heavy gas.

Thus, the mentioned above methods for adding boron during discharge in the PF device to obtain a well-compressed pinch plasma column with hydrogen and boron ions do not seem to be optimal.

So, instead of these methods, we propose to use laser ablation from the boron sample, which is placed in the selected area of the PF anode. This method allows obtaining a boron plume near the anode axis of PF during imploding of hydrogen current sheath in the PF device, which in turn allows obtaining a boron-hydrogen pinch plasma column. Thus, one can expect that in the PF conditions, the fusion reaction of hydrogen and boron nuclei will take place.

Here we present estimates indicating that laser ablation of the boron sample during the normal PF operation is able to generate boron-hydrogen pinch plasma column in which the ${ }^{11} \mathrm{~B}(\mathrm{p}, \alpha) \alpha \alpha$ reaction take place with a yield, high enough to register and measure the alpha particles. Unlike the other approaches, we do not intend to address a potential possibility of maximum fusion gain achievement with this reaction, but we rather want to demonstrate future experimental possibilities to perform aneutronic fusion in the PF device.

\section{Formulation of the Problem}

The idea of the proposed experiment of generating dense and high temperature boron-hydrogen pinch plasma in the PF device is presented in Fig. 1. This idea is based on the general assumption that with the help of the hydrogen plasma layer formed in the PF discharge, you can compress a boron cloud that has been injected with a laser beam from a target placed in the PF anode. Thus, at the end of this process, a dense and high-temperature pinch plasma column with a mixture of hydrogen and boron should be formed. This is the similar to that, when deuterium is injected additionally with a fast electromagnetic valve placed in the anode of PF device and then it compressed by the deuterium plasma layer at the axis to generate the dense high-temperature pinch plasma column [7-9]. Experiments carried out on many PF devices have shown that nuclear DD reactions can take place in such plasma.

Experiments conducted on many PF devices have shown that in such plasma nuclear DD reactions can take place. Thus, it can be expected that, as in the case of deuterium injection through a fast valve, dense and high-temperature hydrogen-boron plasma created by the way as shown in Fig. 1 will be the source of ${ }^{11} \mathrm{~B}(\mathrm{p}, \alpha) \alpha \alpha$ nuclear reaction.

This means that to create homogeneous and compressed plasma column containing ions of hydrogen and boron, a boron cloud must be inject during the development of the discharge in PF (see Fig. 1). In the case of PF-24, the discharge is about 1.5-2.0 $\mu$ s from the electric gas breakdown (see Fig. 1a) and the formation of the plasma layer (PL) (see Fig. 1b), until the PL reaches the anode axis (see Fig. 1c), depending on the pressure of the working gas in the chamber. Here it should be taken into account that the duration of the laser pulse is much shorter, and in our case is 18 ns. Thus, the injection moment, should be chosen as to allow free expansion of the boron plasma to a volume ensuring density of the boron cloud compatible with the density of hydrogen. In this way, a compressed, relatively homogeneous, plasma column will be created, in which phenomena leading to the nuclear ${ }^{11} \mathrm{~B}(\mathrm{p}, \alpha) \alpha \alpha$ reaction may begin to develop.

In many experiments carried out on PF systems with deuterium, it was shown that the main emission of neutrons from the $d(d, n)^{3} \mathrm{He}$ reaction occurs during the development of instability $\mathrm{m}=0$ in the plasma column. As a result of this instability, a neck of compressed plasma appears, with the final radius equal to the thickness of the electric current skin layer. In the next moment, the electric current inside this radius decreases rapidly with a relatively small 


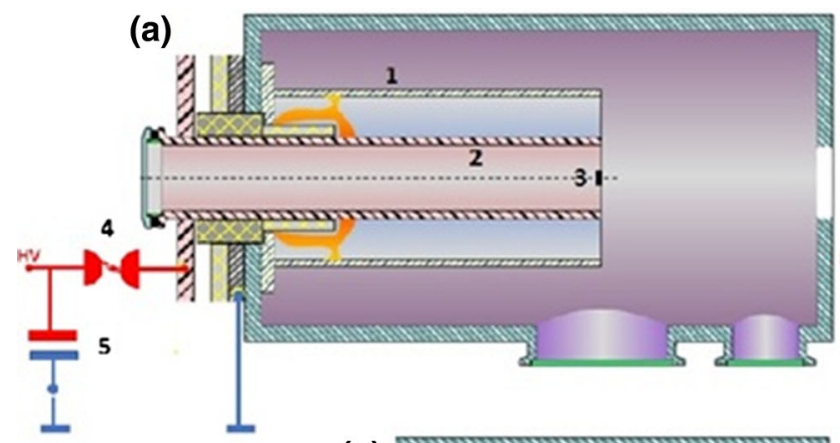

(c)

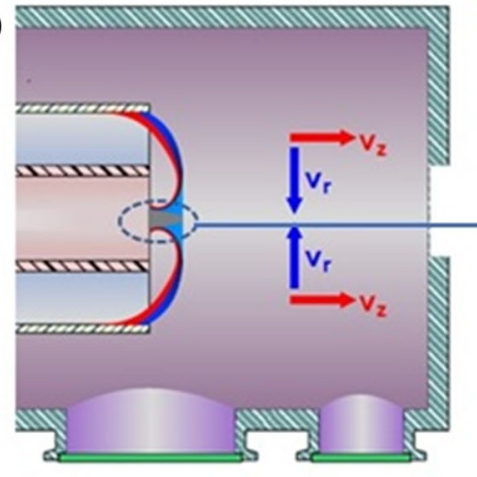

Fig. 1 Idea of the laser ablation experiment in the Plasma-Focus device. a Phase of the breakdown and hydrogen plasma layer (PL) formation, $\mathbf{b}$ phase of the run-down (the acceleration of PL) and moment of laser beam (LB) interaction with target, $\mathbf{c}$ phase of the

change in the total current in the PF system, measured by the Rogowski coil [12]. Then, very fast loss of the conductivity in the pinched plasma, accompanied by a decrease in the plasma density in the neck region is observed, with a simultaneous slight change of the total electric current flowing in the PF system. At the same time, the impulse of electric voltage is observed. It shows, that a flow of the electric current changes its nature in the pinched plasma. It means, that in the area of the neck region the electric current is maintained first by the fast electron beam and then after stopping the electrons in the magnetic field, by the fast ion beam. The fast ion beams are launched from the region of instability (plasma diode) into the residual plasma target in the PF and there they can generate the fusion reactions of the ${ }^{11} \mathrm{~B}(\mathrm{p}, \alpha) \alpha \alpha$.

Taking this into account, it is possible to estimate the number of ${ }^{11} \mathrm{~B}(\mathrm{p}, \alpha) \alpha \alpha$ reactions in the $\mathrm{PF}$ system using the following formula:

$Y_{n}=\left[N_{H} v_{H} \sigma_{H B}\left(E_{H}\right) n_{p l B}+N_{B} v_{B} \sigma_{H B}\left(E_{B}\right) n_{p l H}\right] V_{t p l} \tau_{p l}$

where $N, v$ are the density and a velocity of ions accelerated in the plasma diode created as a result of breaking the electric current in the $m=0$ instability area. $\sigma_{H B}$-the ${ }^{11} \mathrm{~B}(\mathrm{p}, \alpha) \alpha \alpha$ fusion cross-section; $E$ - the kinetic energy of accelerated ions; $n_{p l}$-density of ions in the residual plasma in the PF system and $V_{t p l}, \tau_{p l}$ are the volume of the
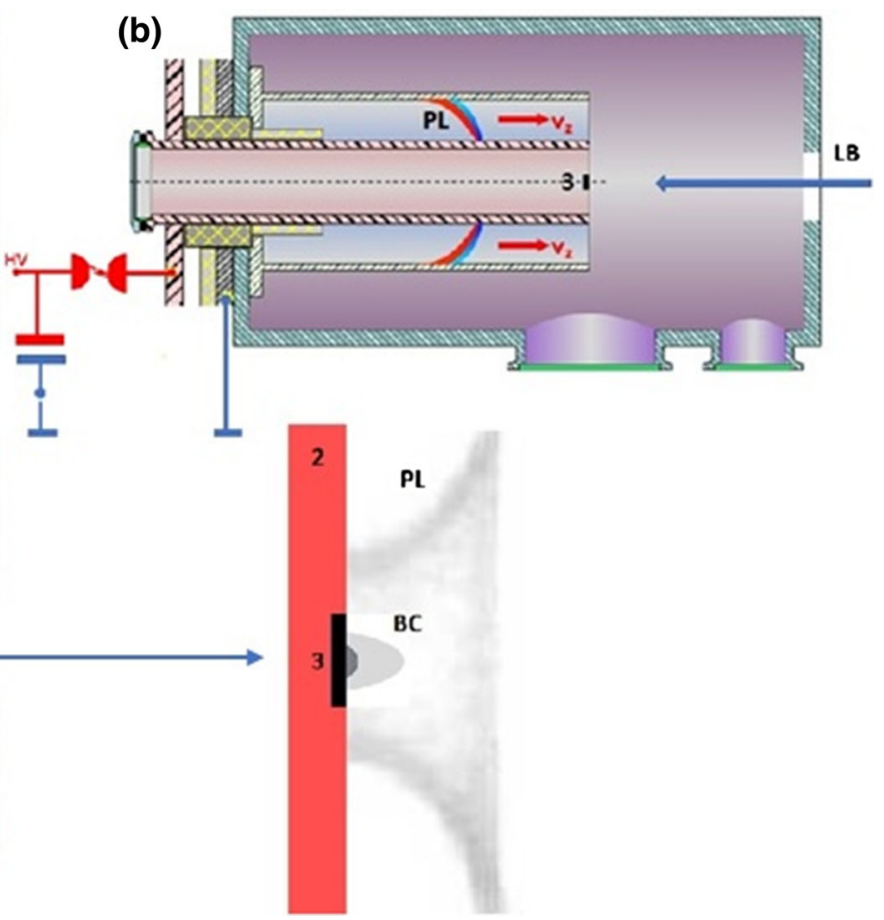

radial compression of PL and free expansion of the boron plasma cloud (BC). 1-the cathode pf Plasma-Focus device, 2-the anode of PF, 3-the boron target, 4-the spark gaps, 5-the condenser bank

residual plasma target and the lifetime of this plasma target, respectively. Indices H, B refer to hydrogen and boron, respectively. Hereafter, it is assumed that the hydrogen and boron ions accelerated in the area of the plasma diode do not interact with each other.

Based on the model presented in [13] one can express the electric field accelerating ions during the disruption of the electric current, by means of the following formula:

$\varepsilon_{z}=-\frac{d I / d t}{c^{2}} K_{0}(x)$

where $K_{0}$-the modified second order Bessel function, $x=$ $\left(r / R_{0}\right)^{2} / 2$ and $R_{0}=\sqrt{I_{0} / 2 \pi n_{e o} e c}$, here $c$-the speed of light, $e$-elementary charge, $I_{0}$ - the value of the electric current at the moment of breaking, $n_{\mathrm{eo}}$-residual concentration of electrons in the $m=0$ instability area. Thus, the momentum of the accelerated ions in the instability region can be determined by the formula:

$\frac{d p}{d t}=Z e \varepsilon_{z}=-Z e \frac{d I / d t}{c^{2}} K_{0}(x)$

where $Z$ is a charge number of the accelerated ions. Solving the Eq. (3) with the value of the electric field expressed in Eq. (2) and taking the asymptotic form of the Bessel function for $x \ll 1$, we can describe the velocity of the accelerated beam ions by the equation: 
$p=-\frac{Z e K_{0}(x)}{c^{2}} \int_{0}^{T} \frac{d I}{d t} d t \cong \frac{Z e I_{0}}{c^{2}} \ln \left[\frac{4}{1.78}\left(\frac{R_{0}}{r}\right)^{2}\right]$

In Eq. (4), the factor 1.78 comes from the Euler's constant. For further considerations, it was assumed that $r$ is equal to the skin depth $\delta_{s k} \cong I_{0} v_{r} /(d I / d t)_{0}, v_{r}$-average velocity of the plasma sheath before breaking the electric current.

In Eq. (4) it was assumed that the electric conductivity current at the moment of breaking the plasma column decreases in the area of instability, from the value of $I_{0}$ to zero. Due to the fact that the electron plasma temperature is in the order of 200-300 eV [14], it can be further assumed that the boron particles are completely ionized. So, for hydrogen $p_{\mathrm{H}}=p$ with $Z=1$ [see Eq. (4)] and for boron $p_{B}=Z_{\mathrm{B}} p_{\mathrm{H}}$. Based on Eq. (4), the energy of the proton and boron ion beams can be calculated, respectively.

The ion number density of accelerated ions in the plasma diode is a sum of two ion species hydrogen and boron $N_{\mathrm{i}}=N_{H}+N_{B}$. If the mixture ratio $\gamma$ is defined to be a ratio of the number density of the hydrogen to the total number of the ion density, one obtains $\gamma \equiv N_{\mathrm{H}} / N_{i}$ and ratio of the number density of the boron to the total ion density is given by $\left(N_{B} / N_{i}\right)=1-\gamma$. In the rest of the plasma column-plasma target, the total number of ion density $n_{p l i}=n_{p l H}+n_{p l B}$ and the mixture ratio $n_{p l H} / n_{p l i}$ can be specified similarly. For simplicity, we assume that $\left(n_{p l H} / n_{p l i}\right)=\gamma$.

After simple algebraic transformations, Eq. (1) now takes the form:

$Y_{n}=\left[\sigma_{H B}\left(E_{H}\right)+\frac{Z_{B} M_{H}}{M_{B}} \sigma_{H B}\left(E_{B}\right)\right] \frac{\gamma(1-\gamma)}{M_{H}} n_{p l i} N_{i} p_{H} V_{t p l} \tau_{p l}$

here $M_{H}, M_{B}$ are the masses of the hydrogen and boron, respectively.

The incoming particle flux $N_{i} p_{H}$ can be determined from:

$j_{i}=\frac{e}{M_{H}}\left[\gamma+\frac{Z_{B}^{2} M_{H}}{M_{B}}(1-\gamma)\right] N_{i} p_{H}$

where $j_{i}$ is the ion current emission density into a plasma diode. This current can be determined by known ChildLangmuir formula:

$j_{i} \cong \frac{1}{9 \pi}\left(\frac{2 e}{M_{H}}\right)^{1 / 2}\left[1+\left(\frac{Z_{B} M_{H}}{M_{B}}\right)^{1 / 2}\right] \frac{\phi^{3 / 2}}{l_{z n}^{2}}$

where $\phi$ denotes the electric potential in a plasma diode, while $l_{z n}$ is a neck dimension of the $\mathrm{m}=0$ instability. So, the final formula for the $N_{\mathrm{i}} p_{\mathrm{H}}$ is as follows:
$N_{i} p_{H}=\frac{1}{9 \pi}\left(\frac{2 M_{H}}{e}\right)^{1 / 2} \frac{1+\left(\frac{Z_{B} M_{H}}{M_{B}}\right)^{1 / 2}}{\gamma+\frac{Z_{B}^{2} M_{H}}{M_{B}}(1-\gamma)} \frac{\phi^{3 / 2}}{l_{z n}^{2}}$

Further, as it can be seen from Eq. (5), the total number of ion densities $n_{p l i}=n_{p l H}+n_{p l B}$ in the residual plasma should be determined in order to estimate the amount of

${ }^{11} \mathrm{~B}(\mathrm{p}, \alpha) \alpha \alpha$ reactions in the PF system.

For this purpose, the mass released by the laser from the surface of the boron sample placed in the chamber of the PF device was measured. Next, the size of the vaporplasma plume expansion was estimated. This enables to calculate the total number of boron ion densities $n_{\mathrm{OB}}$ that can be compressed by the hydrogen plasma layer. The total number of the hydrogen ion densities $n_{0 \mathrm{~B}}$ can be determined by knowing the hydrogen pressure in the PF chamber, based on the ideal gas equation.

\section{Measurement of Ablated Mass from a Boron Sample Placed in the PF Chamber}

To perform an experiment of proton-boron plasma pinch formation in the PF-24 device, in the first stage a setup for experimental boron ablation using a pulsed laser was prepared. This preliminary experiment allowed to solve the following issues:

- Whether the laser used may cause self-breakdown of the working gas in the PF chamber in front of the sample;

- Measurement of ablated mass in this experiment;

- Comparison of the measured and estimated ablation mass.

A Nd:YAG laser (Lotis TII, LS-2147) was used in this experiment. The applied laser beam has the following parameters: Gaussian profile, time of impulse $18 \mathrm{~ns}$, wavelength $1064 \mathrm{~nm}$ and diameter $8 \mathrm{~mm}$. In the case of the Gaussian beam, the radiation intensity smoothly decays from the central point to the edge of the laser spot. The energy of the laser pulse could be changed from 400 to $900 \mathrm{~mJ}$. In order to focus the beam, the lens with an effective focal length of $125 \mathrm{~mm}$ was used. A lens dedicated to the infrared radiation was selected. The crystalline boron sample of $99.6 \%$ purity with a polished surface was located on a small piece of laboratory glass and glued with epoxy glue. Thus, the prepared sample was placed $122.7 \mathrm{~mm}$ from the lens in the PF vacuum chamber. The length of $122.7 \mathrm{~mm}$ is the lower focal length i.e. the distance between the flat surface of the lens and the focal point. During laser shots, the gas pressure in the vacuum chamber was about 0.01-0.02 mbar. 
Two series of experiments were performed. The first for verify deuterium self-breakdown with a laser beam in the pressure range of 1-3 mbar and the second for estimate quantity of ablated mass. During the first series no selfbreakdown was found in this pressure range, a crater appeared regularly on the target in the place of the focused laser beam.

The experimental setup for the measurement of ablated mass from the boron sample placed in the PF vacuum chamber is shown in Fig. 2.

In this experimental setup, 1 and 10 laser shots were made, each with the laser energy $E_{\mathrm{L}}=707 \mathrm{~mJ}$.

The ablation mass was measured indirectly using an optical profilometer: Filmetrics 3D Profiles. The result from the profilometer is shown in Fig. 3. Based on the profiles for 1 and 10 shots, the volume of the removed material and then its mass were estimated. In order to estimate the volume of the removed mass, it was assumed that the crater has the shape of a frustum of a cone.

As a result, we obtained $0.7( \pm 0.4) \mu \mathrm{g}$ of ablated boron mass per shot. Hence, in further calculations, we assumed that ablated mass per one shot is equal to $0.7 \mu \mathrm{g}$. Based on the measured ablated mass, it is possible to estimate the size and density of the boron vapor-plasma plume near the axis of the PF anode.

\section{Estimation of the Size and Density of Boron Vapor-Plume Plasma}

In the case of laser nanosecond pulses, the radiation heats the sample. This process begins with the absorption of the laser energy in the sample. The absorption is associated with the conduction of band electrons, thermal conductivity, and electron-lattice collisions. Finally, the laser beam with a several-nanosecond pulse produces a vapor-plasma plume on the sample surface which is located on the anode

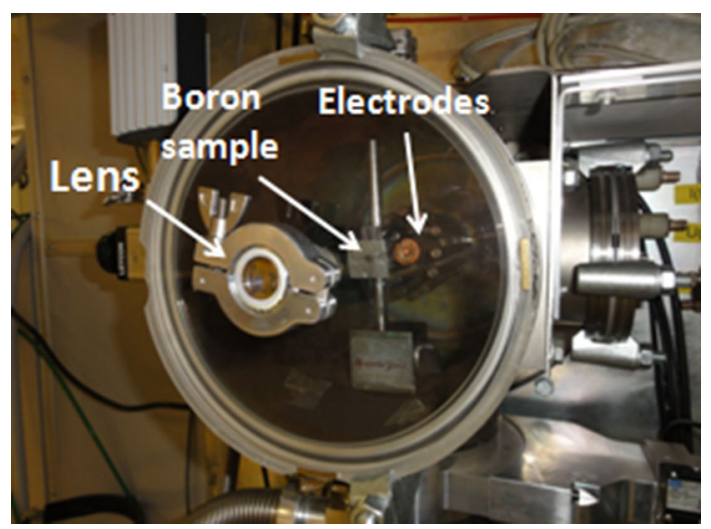

Fig. 2 The vacuum chamber of the PF device with a boron sample placed inside and a specially prepared vacuum flange with a lens of the PF device (see Fig. 4). The initial plasma evaporated material (plasma plume) was created at $t=0$, after the end of laser pulse near the surface of the sample. We assumed that the plasma cloud has elliptical symmetry during its expansion for $t>0$, as it was assumed in [15].

Based on the analytical solutions presented in [15] for $\kappa=c_{p} / c_{v}=5 / 3$, the dimensions of the plasma cloud can be estimated using the formulas (see Fig. 4):

$b(t)=r_{0} \sqrt{3\left(\frac{t}{t_{0}}\right)^{2}+\left(\frac{z_{0}}{r_{0}}\right)^{2}+2}, a(t)=\frac{b(t)}{\sqrt{2}}$

The initial radius of the plume $r_{0}$ can be approximated by the radius of the laser spot. The height of the plume in the $\mathrm{z}$-direction is about $z_{0}=v_{s} \tau$, where $v_{s}$ is the velocity of sound and $\tau$ is the laser pulse duration. In our experiments typical value of the $r_{0} \cong 500 \mu \mathrm{m}$ are obtained. Assuming that the evaporated material can be described as an ideal gas with constant adiabatic exponent $\kappa=c_{p} / c_{\nu}=5 / 3$, the values are $z_{0} \cong 1.27 \times 10^{-3}(T / A)^{1 / 2} \tau \mathrm{cm}$, where $T$ is the plasma temperature in $\mathrm{eV}, A$ is atomic mass number and $\tau$-laser pulse duration in nanosecond. The characteristic time scale for expansion $t_{0}$ is

$t_{0}=\frac{r_{0}}{\sqrt{(5 \kappa-3) E / M_{a}}}$

where $E$ - the initial energy of the plasma plume and $M_{a}$ is the ablatedmass during laser shot.

In this approximation the initial energy in the plasma plume can be estimated by the formula:

$E=E_{a}-\left(E_{m}+E_{v}\right)$.

here $E_{a}$ is the laser energy absorbed by the sample. $E_{m}$ is roughly given by the energy needed to melt the laser spot area to a depth of $l_{t h}$ and it is given as follows:

$E_{m}=\rho\left[\mathrm{C}\left(T_{m}-T_{i}\right)+L_{m}\right] l_{t h} S_{f}$,

where $\rho$ is a sample density, $\mathrm{C}$ and $L_{m}$ are heat capacity and latent heat for melting (per unit mass of material), $T_{m}, T_{i}$ melting and initial temperature, respectively. $l_{t h}$ is given by expression $l_{t h} \cong \sqrt{2 D \tau_{p}}$ and $S_{f}$ is the laser spot area. $D$ is the heat diffusion coefficient and $\tau_{p}$ is the pulse duration. $E_{v}$ is the energy needed for the vaporization of the melting material of sample and is given as:

$E_{v}=M_{a} L_{v}$.

where $L_{v}$-latent heat for vaporization (per unit mass of material) and $M_{a}$ as previously is the ablated mass.

Now, using the formula (9), assuming that the plasma cloud has elliptic symmetry, the volume of this cloud was determined, according to the expression:

$V(t)=\frac{2}{3} \pi a^{2}(t) b(t)$ 

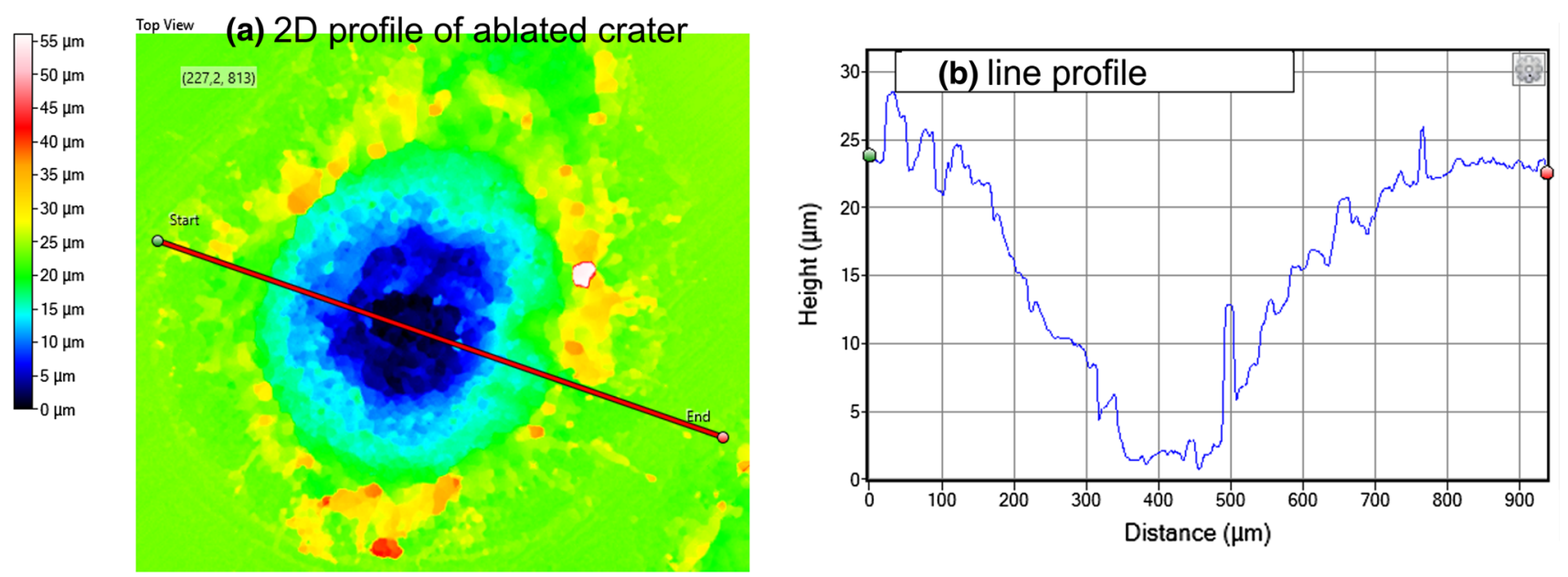

Fig. 3 The profiles (a 2D profile; $\mathbf{b}$ line profile) of the crater created in the boron sample after ten laser pulses

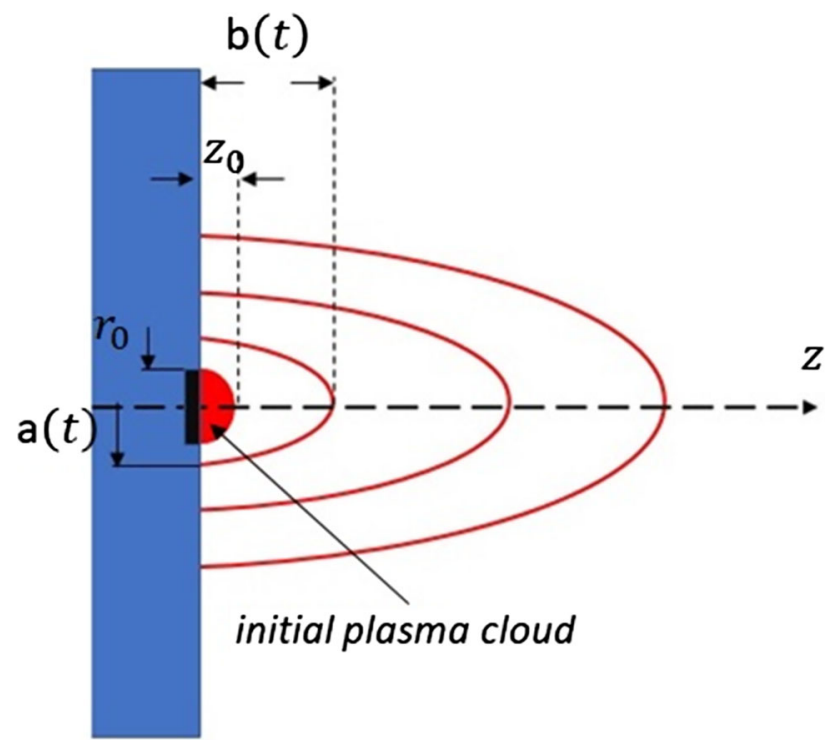

Fig. 4 Scheme of the plasma cloud expansion from the anode of the PF device

If we assume, for the sake of simplicity, that boron ions are uniformly distributed within this volume, their concentration can be determined from the following expression:

$n_{0 B}=N_{A} \frac{M_{a}}{M_{m} V(t)}$,

where $-N_{A}$ the Avogadro number, $M_{m}$-molar mass of boron, $M_{a}$ - the mass of ablated boron, $V(t)$ - the volume of plasma cloud at time $t$, expressed by (9).

\section{Results and Discussion}

This section presents the $Y_{n}$ estimation for future ablative experiments on the PF-24 device using the Nd:YAG laser described above.

As it can be seen from Eq. (5), the number of ${ }^{11} \mathrm{~B}(\mathrm{p}$, $\alpha) \alpha \alpha$ reactions in the $\mathrm{PF}$ system depends on the energy of accelerated $\mathrm{H}$ and $\mathrm{B}$ ions, their stream $N_{i} p_{H} / M_{H}$, the density and composition of the plasma target as well as on its volume and lifetime. The estimations assume that the energy of ions ( $\mathrm{p}$ and B) accelerated during the $m=0$ instability is defined and can be determined using Eq. 4 in the following form:

$E_{H} \cong 4.82 \times 10^{2} I_{0}^{2} \ln \left[2.25\left(\frac{R_{0}}{\delta_{s k}}\right)^{2}\right][\mathrm{keV}]$

here $\quad R_{0} \cong 5.76 \times 10^{6} \sqrt{I_{0} / n_{e o}}[\mathrm{~cm}] \quad$ and $\quad \delta_{s k} \cong I_{0} v_{r} /$ $(d I / d t)_{0}[\mathrm{~cm}] . I_{0}$-is expressed in MA, $n_{e o}$ in $\mathrm{cm}^{-3}$, and $v_{r}$ in $\mathrm{cm} / \mathrm{s}$. The expression for boron ion energy has the following form according to Eq. (4), where $Z_{B}$ is the charge of the accelerated boron ion. It was assumed that $Z_{B}=5$, i.e. the boron atom was completely ionized.

Figure 5 shows the dependence of the energy of accelerated hydrogen ions with respect to the current $I_{0}$ for different values of the current derivative. It was assumed that the concentration of "residual" plasma $n_{e o} \approx$ $10^{13} \mathrm{~cm}^{-3}$, and $v_{r} \approx 10^{7} \mathrm{~cm} / \mathrm{s}$.

The range of numerical values of $I_{0}$ was selected considering future experiments on the PF-24 device.

The assumption was made that the mixture ratio $\gamma$ is conserved during the whole process and is the same in both the $m=0$ instability and the plasma target. In addition, we assumed that the total concentration of ions in the pinch is $n_{p l} \approx 100 n_{0}$, where $n_{0}=n_{0 H}+n_{0 B}$ - the total, initial concentration of ions compressed by the current sheath. 


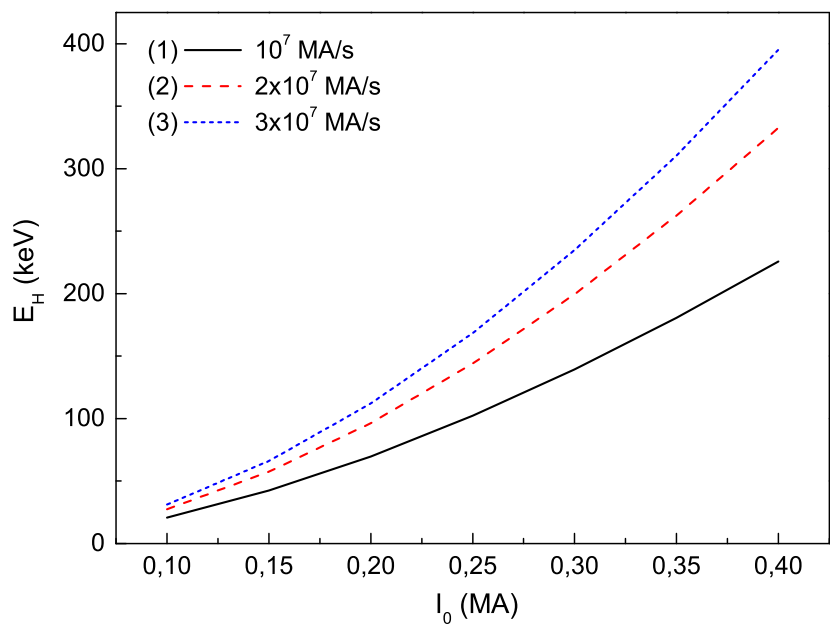

Fig. 5 Energy of hydrogen ions as a function of the current $I_{0}$, for different derivative values: (1) solid line $-10^{7} \mathrm{MA} / \mathrm{s}$, (2) dashed line $-2 \times 10^{7} \mathrm{MA} / \mathrm{s}$, (3) dotted line-3 $3 \times 10^{7} \mathrm{MA} / \mathrm{s}$

Here $n_{0 H}$ depends on the initial pressure of hydrogen filling the PF-24 vacuum chamber, and $n_{0 B}$ on the ablated boron mass from the sample, depending on the laser energy and the characteristic expansion time $t_{0}$ [Eq. (10)]. Figure 6a shows the $n_{0 B} / n_{0 H}$ ratio dependent on $t_{0}$ for two different working gas pressures. Figure $6 \mathrm{~b}$ shows the change in time of the dimensions of the plasma cloud in the radial and axial direction, with respect to the anode face of the PF-24. Shown in Fig. 6 dependencies were obtained for $M_{a}=0.7 \mu \mathrm{g}$, the laser energy $\mathrm{E}=707 \mathrm{~mJ}, r_{0} \cong 500 \mu \mathrm{m}$ (see Fig. 4), and the initial plasma temperature $T=1 \mathrm{eV}$.

The knowledge of time $t_{0}$ and plasma cloud expansion conditions allows to determine the synchronization between the moment of laser ablation completion and the time of plasma sheath compression in the PF-24 device.

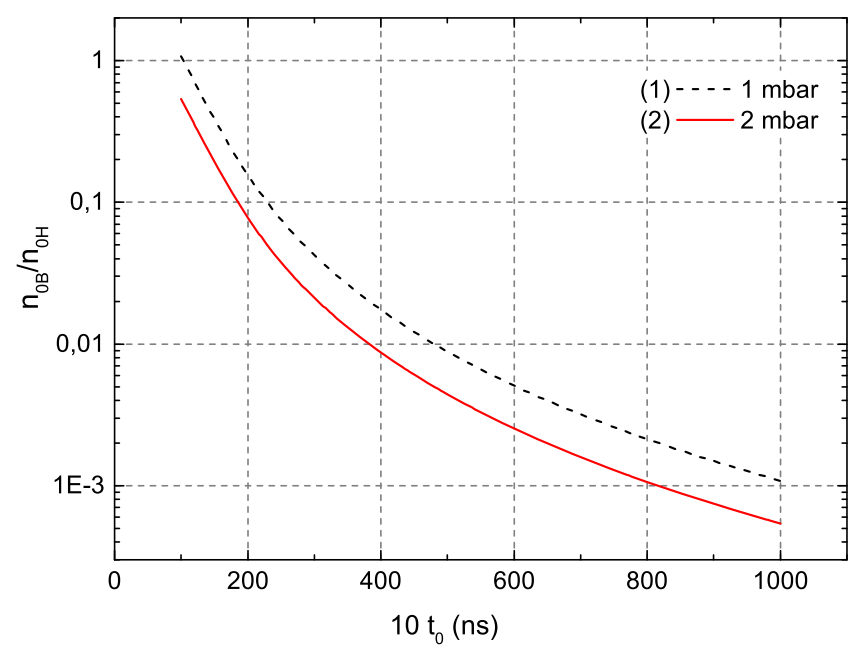

The dependence of the stream of accelerated ions on the voltage pulse in the PF system is shown in Fig. 7, where $\Psi=N_{i} p_{H} / M_{H}$ (Eq. 8). $l_{z n}=0.5 \mathrm{~cm}$ was assumed for the calculations.

The range of the voltage pulse values is characteristic for the PF-24. To estimate the number of ${ }^{11} \mathrm{~B}(\mathrm{p}, \alpha) \alpha \alpha$ reaction in the PF-24, the cross section was taken from [16].

In the case of PF-24, the energy range for hydrogen ions is $50-300 \mathrm{keV}$ and for ${ }^{11} \mathrm{~B} 100-900 \mathrm{keV}$, as shown in Fig. 8 .

In order to estimate $Y_{n}$, we assume values $E_{H}, E_{B}$, for $I_{0}=0.35 \mathrm{MA}$, with the value of current derivative $3 \times$ $10^{7} \mathrm{MA} / \mathrm{s}$ (see Fig. 8), and the value of stream $\Psi$ was calculated for $\phi=250 \mathrm{kV}$, with hydrogen pressure in the PF-24 chamber equal to $1 \mathrm{mbar}$ and $n_{0 B} / n_{0 H}=0.27$. In addition, with a good approximation, it can be assumed that the total number of ions in the plasma target for the assumed pressure values and $n_{0 B} / n_{0 H}$, will be approximately equal to $n_{p l} \approx 7 \times 10^{18} \mathrm{~cm}^{-3}$. Taking the above numerical values and assuming the volume of the plasma target $V_{t p l} \sim 3-4 \mathrm{~cm}^{3}$ and its life-time $\tau_{p l} \sim 50 \mathrm{~ns}$, it can be estimated that the number of ${ }^{11} \mathrm{~B}(\mathrm{p}, \alpha) \alpha \alpha$ reactions occurring in the PF-24 system in experiments with laser boron sample ablation can range from $10^{7}$ to a maximum of $10^{8}$ reactions per discharge. This number of reactions allows the detector to register $\alpha$ particles emitted into a full solid angle, provided that the detector with appropriate sensitivity will not be too far away from the emission source.

The numerical values of the electrical parameters used to calculate the ${ }^{11} \mathrm{~B}(\mathrm{p}, \alpha) \alpha \alpha$ reaction were adopted from experiments on the PF-24 system with deuterium as a working gas. The other parameters used in the model were

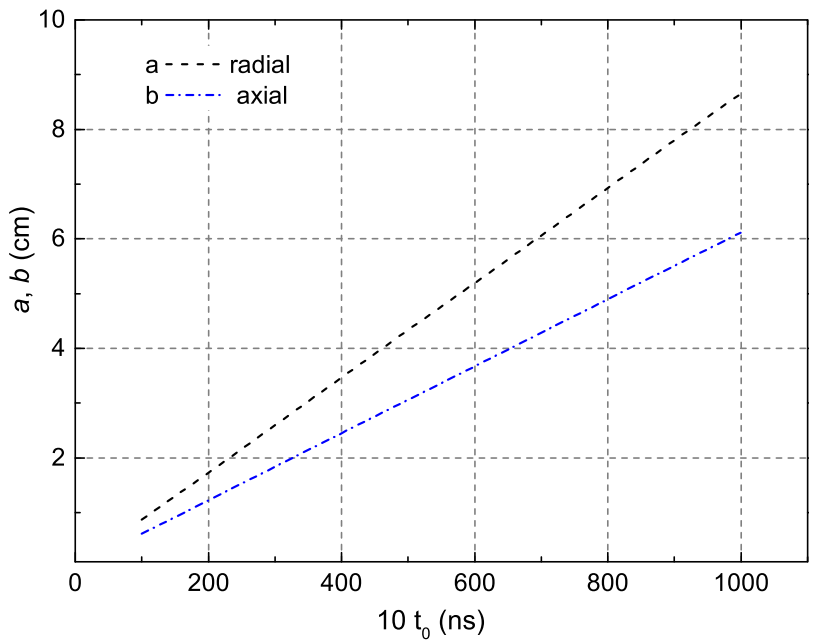

Fig. 6 a Ratio $n_{0 B} / n_{0 H}$ for different hydrogen pressures (1) dashed line-1 mbar, (2) solid line-2 mbar. b Radial (a) and axial (b) dimensions of the plasma cloud for hydrogen pressure $1 \mathrm{mbar}$ and $M_{a}=1.2 \mu \mathrm{m}$ 


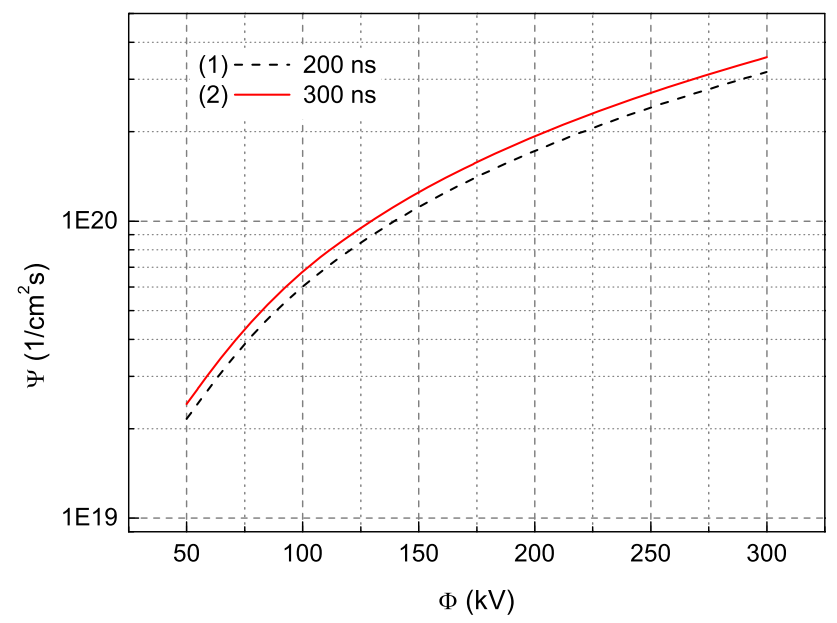

Fig. 7 The stream of accelerated ions $\Psi$ as a function of the voltage pulse in the PF system. The parameter $\left(n_{0 B} / n_{0 H}\right)$ was taken from Fig. 6a for (1) dashed line-200 ns and (2) solid line $-300 \mathrm{~ns}$

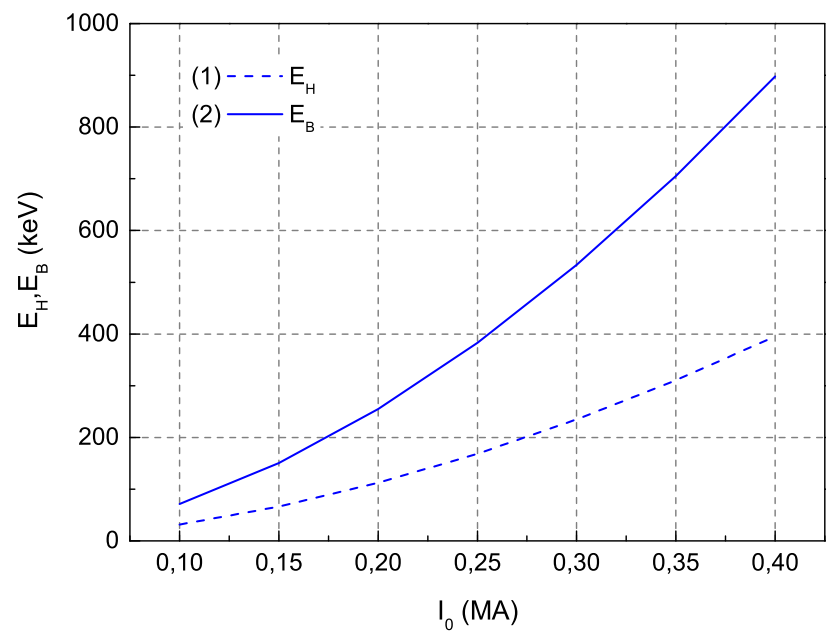

Fig. 8 The energy of hydrogen and boron ions as a function of $I_{0}$, for the given value of the current derivative $3 \times 10^{7} \mathrm{MA} / \mathrm{s}$; (1) dashed line $-E_{H}$, (2) solid line $-E_{B}$

chosen somehow arbitrarily, however, in accordance with the parameters measured or estimated for other PF systems. Another issue requiring consideration would be to supplement the plasma diode model in such a way that obtaining the energy distribution of accelerated ions would be possible. The ablation mass was determined experimentally with a boron sample placed in the PF chamber based on the measurement of the crater size. The experiment showed that during each single laser shot with a given energy, it released approximately the same amount of mass from the boron sample. In addition, the dynamics of the boron plasma cloud did not take into account the presence of working gas in the PF-24 chamber.

\section{Conclusions}

The considerations and analyses carried out in this article can be summarized by the following conclusions:

1. The estimated number of ${ }^{11} \mathrm{~B}(\mathrm{p}, \alpha) \alpha \alpha$ nuclear reactions in the PF system, allows concluding that in future experiments on the PF-24 device using the Nd:YAG laser, it will be possible to choose $\alpha$ particle detectors, plan their proper placement near the emission source and measure the flux of alpha particles, with sufficient accuracy.

2. The models presented here give the possibility to estimate the emission of alpha particles based on the measured electrical parameters (current, current derivative, the voltage on the probe) of the PF-24 system. This allows rough experimental verification of the adopted assumptions and approximations in the developed models.

3. Estimation of the dynamics of the plasma cloud expansion caused by ablation gives the possibility of correlating the moment of laser beam generation with the time course of acceleration and plasma sheath compression in the PF-24 system.

4. The authors are aware that some approximations used in the course of the performed estimations and the parameters adopted for the calculation may be inaccurate. This applies, for example, to the assumption that accelerated ions have the same specific energy, not some energy distribution.

The presented idea of doping plasma in the PF systems by means of laser ablation seems to be attractive for two reasons. First, it provides the opportunity to the operator to change the plasma density regardless of the working gas pressure, making the laser ablation method competitive with systems using a fast gas valve. Secondly, it enables plasma doping with elements that do not occur in the gaseous state under normal conditions. This issue, in turn, may be of great importance for research related to X-ray spectroscopy of plasma doped with large $\mathrm{Z}$ elements.

Open Access This article is distributed under the terms of the Creative Commons Attribution 4.0 International License (http://creative commons.org/licenses/by/4.0/), which permits unrestricted use, distribution, and reproduction in any medium, provided you give appropriate credit to the original author(s) and the source, provide a link to the Creative Commons license, and indicate if changes were made. 


\section{References}

1. W.M. Nevins, J. Fusion Energ. 17, 25 (1998)

2. D.C. Moreau, Nucl. Fusion 17, 13 (1977)

3. V.S. Belyaev, A.P. Matafanov, V.I. Vinogradov et al., Phys. Rev. E 72, 026406 (2005)

4. N. Rostoker, M.W. Binderbauer, H.J. Monkhorst, Science 278, 1419 (1997)

5. H. Hora, G.H. Miley, M. Ghoranneviss et al., Opt. Commun. 282, 4124 (2009)

6. E.J. Lerner, S.K. Murali, A. Haboub, J. Fusion Energ. 30, 367 (2011)

7. H. Schmidt, M. Sadowski, L. Jakubowski et al., Plasma Phys. Control. Fusion 36, 13 (1994)

8. M. Milanese, R. Moroso, J. Pouzo, J. Phys. D Appl. Phys. 31, 85 (1998)

9. J. Stanislawski, J. Baranowski, M. Sadowski et al., Nukleonika 46(Supplement 1), 73 (2001)
10. M. Scholz, L. Karpinski, W. Stepniewski et al., Phys. Lett. A 262, 453 (1999)

11. V.P. Vinogradova, M.A. Karakin, V.I. Krauz et al., Plasma Phys. Rep. 32, 642 (2006)

12. K.N. Mitrofanov, V.I. Krauz, P. Kubes et al., Plasma Phys. Rep. 40, 623 (2014)

13. B.A. Trubnikov, Fizika Plazmy 12, 468 (1986)

14. J. Abdallah Jr., R.E.H. Clark, A.Y. Faenov, L. Karpinski, S.A. Pikuz, V.M. Romanova, M. Sadowski, M. Scholz, A. Szydlowski, J. Quant. Spectrosc. Radiat. Transf. 62, 85 (1999)

15. S.I. Anisimov, D. Bäurele, B.S. Luk'yanchuk, Phys. Rev. B 48, 12076 (1993)

16. W.M. Nevins, R. Swain, Nucl. Fusion 40, 865 (2000)

Publisher's Note Springer Nature remains neutral with regard to jurisdictional claims in published maps and institutional affiliations. 